\title{
ARTICLE
}

Received 18 Jul 2012 | Accepted 25 Sep 2012 | Published 23 Oct $2012 \quad$ DOl: 10.1038/ncomms2162

\section{Emergent quantum confinement at topological insulator surfaces}

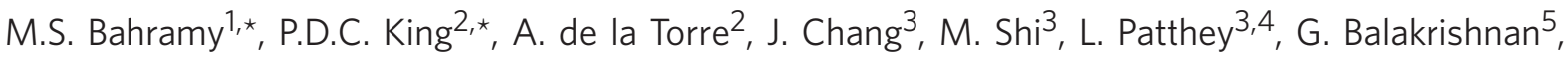 \\ Ph. Hofmann ${ }^{6}$, R. Arita ${ }^{1,7}$, N. Nagaosa ${ }^{1,7,8} \&$ F. Baumberger ${ }^{2}$
}

Bismuth-chalchogenides are model examples of three-dimensional topological insulators. Their ideal bulk-truncated surface hosts a single spin-helical surface state, which is the simplest possible surface electronic structure allowed by their non-trivial $Z_{2}$ topology. However, real surfaces of such compounds, even if kept in ultra-high vacuum, rapidly develop a much more complex electronic structure whose origin and properties have proved controversial. Here we demonstrate that a conceptually simple model, implementing a semiconductor-like band bending in a parameter-free tight-binding supercell calculation, can quantitatively explain the entire measured hierarchy of electronic states. In combination with circular dichroism in angle-resolved photoemission experiments, we further uncover a rich three-dimensional spin texture of this surface electronic system, resulting from the non-trivial topology of the bulk band structure. Moreover, our study sheds new light on the surface-bulk connectivity in topological insulators, and reveals how this is modified by quantum confinement.

\footnotetext{
${ }^{1}$ Correlated Electron Research Group (CERG), RIKEN-ASI, Wako, Saitama 351-0198, Japan. ${ }^{2}$ SUPA, School of Physics and Astronomy, University of St Andrews, St Andrews, Fife KY16 9SS, UK. ${ }^{3}$ Swiss Light Source, Paul Scherrer Institut, CH-5232 Villigen PSI, Switzerland. ${ }^{4}$ SwissFEL, Paul Scherrer Institut, CH-5232 Villigen PSI, Switzerland. ${ }^{5}$ Department of Physics, University of Warwick, Coventry CV4 7AL, UK. ${ }^{6}$ Department of Physics and Astronomy, Interdisciplinary Nanoscience Center, Aarhus University, 8000 Aarhus C, Denmark. ${ }^{7}$ Department of Applied Physics, University of Tokyo, Tokyo 113-8656, Japan. ${ }^{8}$ Cross-Correlated Materials Research Group (CMRG), RIKEN-ASI, Wako, Saitama 351-0198, Japan. ${ }^{*}$ These authors contributed equally to this work. Correspondence and requests for materials should be addressed to P.D.C.K. (email: philip.king@st-andrews.ac.uk)
} 
T opological insulators are an exotic state of quantum matter, guaranteed to have metallic edge or surface states due to an inverted ordering of their bulk electronic bands ${ }^{1}$. The corresponding topological invariants ${ }^{2}$ dictate that there must be an odd number of such states intersecting the Fermi level between each pair of surface time-reversal invariant momenta. In the most widely investigated bismuth-chalchogenide family of topological insulators, there is just one of these so-called topological surface states (TSSs) creating a single Dirac cone around the Brillouin zone centre ${ }^{3-6}$. These materials are therefore widely regarded ideal templates to realize the predicted exotic phenomena and applications of this TSS. However, exposure to even the minute amount of residual gas in an ultra-high vacuum chamber induces a drastic reconstruction of the surface electronic structure. The Dirac point shifts to higher binding energies, indicating more electron-rich surfaces ${ }^{7-10}$. More importantly, additional pairs of two-dimensional, almost parabolic states emerge in the vicinity of the bulk conduction band ${ }^{7}$, which develop large Rashba-type splittings ${ }^{8}$, whereas new ladders of M-shaped states are created in the original bulk valence bands ${ }^{7,11}$. Given their propensity for formation, these must be considered part of the intrinsic electronic structure of the surface of any realistic topological insulator, for example when exposed to air or interfaced to another material. However, their existence is not predicted by idealized theoretical models of the bulk-truncated surface ${ }^{3}$, and has proved controversial. Recent proposals attribute their formation to a variety of electronic or structural modifications of the crystal host ${ }^{7-9,11-22}$, but to date no single model has been able to simultaneously reproduce the number and binding energies of the experimentally observed electronic states as well as the magnitude of their measured Rashba-type splittings.

In the following, we show that this can be achieved taking account of only a single electronic phenomenon-a near-surface electrostatic potential variation. Such intrinsic electric fields are familiar from surface space-charge regions in conventional semiconductors ${ }^{23,24}$, and are also formed at interfaces where they can be modulated using applied gate voltages. Thus, our findings are not only relevant to understand the free surfaces of generic topological insulators, but also their use in transistor-style or other thin-film device architectures, which may ultimately provide one of the most promising routes towards spintronics. Our study elucidates the crucial interplay of quantum confinement with topological order for such systems, and uncovers a unique spin texture of the resulting electronic states. We consider $\mathrm{Bi}_{2} \mathrm{Se}_{3}$ as a model example, but our computational approach can be freely adapted to virtually all topological insulators, such as other emerging Bi-chalcogenides or the halfHeusler alloys ${ }^{25}$, where we would expect a similarly striking role of band bending at free surfaces, interfaces, or in devices.

\section{Results}

Band-bending-driven quantum confinement. We implement such a band bending scenario (Fig. 1a) within a 120 quintuplelayer tight-binding supercell calculation (see Methods). Without incorporating any band bending, our supercell model yields a single TSS spanning from the bulk valence to conduction bands (Supplementary Fig. S1), in good agreement with both firstprinciples slab calculations ${ }^{3}$ and our experimental measurements of the pristine surface (Supplementary Fig. S2). Additionally including an electrostatic potential variation, we find a hierarchy of electronic states emerges that is in excellent agreement with our experimental angle-resolved photoemission (ARPES) measurements of a doped topological insulator surface (Fig. 1b,c). The effective mass is slightly overestimated in our model, which stems from a small overestimation of this quantity in our bulk band

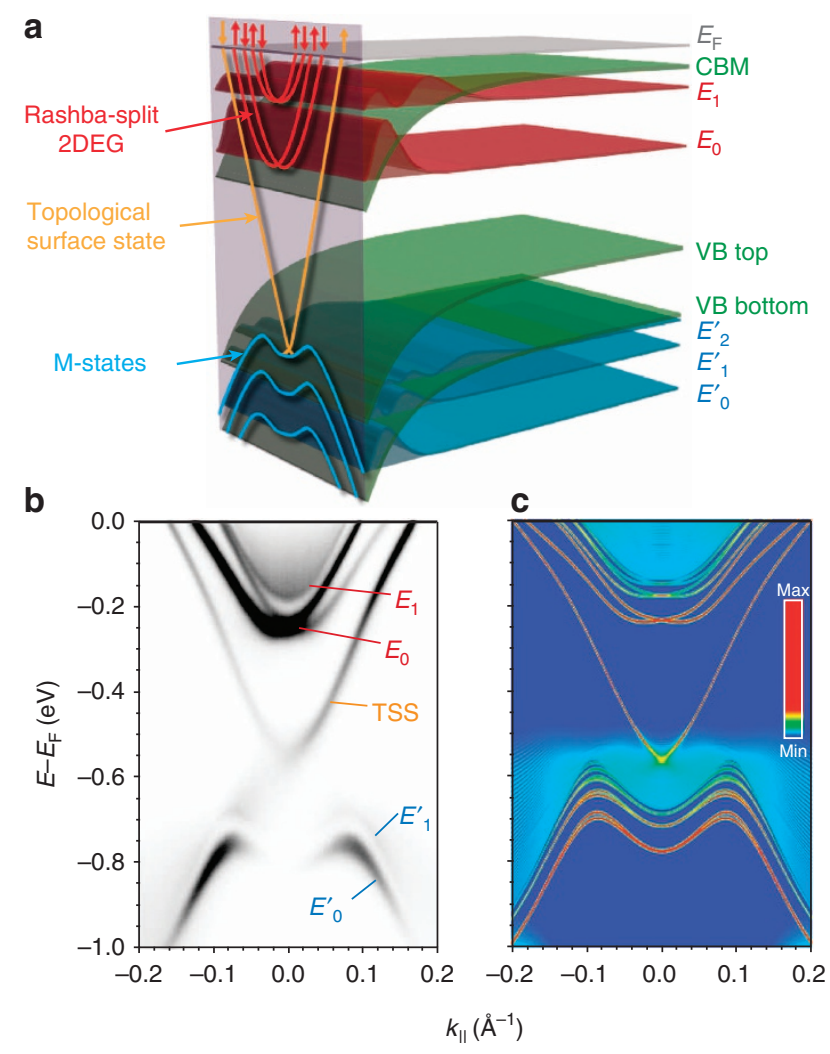

Figure 1 | Band-bending-induced surface electronic structure of $\mathrm{Bi}_{2} \mathrm{Se}_{3}$. (a) A near-surface electrostatic potential variation causes downward band bending of both the conduction band minimum (CBM) and valence band (VB) edges, forming a surface quantum well. It shifts the Dirac point of the TSS (yellow band) further below the Fermi level and causes the conduction band states to be restructured into ladders of Rashba-split two-dimensional subbands, $E_{n}$ (red bands), with envelope wave functions (dark red) peaked close to the surface. A schematic spin texture of these states is shown by the arrows. The finite valence band width provides simultaneous confinement of the valence band states, causing these to also become quantized into ladders of $M$-shaped states, $E_{n}{ }^{\prime}$ (blue bands). These features can all be seen in ARPES measurements of the surface electronic structure (b) and are well described by the surface projection (that is, real-space projection onto the first three quintuple layers) of an $a b$ initio-derived tight-binding model (c) of the ideal $\mathrm{Bi}_{2} \mathrm{Se}_{3}$ bulk structure subject only to a perturbing electrostatic potential close to the surface (false colour scale inset). $k_{\|}$is along the $\Gamma-\mathrm{M}$ direction.

structure calculations. Nonetheless, all other features are well reproduced. This agreement is remarkable, given that we do not adjust any parameters in our calculation. All tight-binding matrix elements are obtained from an ab initio calculation of the bulk electronic structure. The magnitude of the potential change is fixed by the experimentally measured shift of the Dirac point between a pristine-cleaved surface and the $n$-type doped one (see Supplementary Fig. S2), and its functional form follows from a solution of Poisson's equation ${ }^{26}$.

All of the new electronic states observed experimentally arise simply due to the perturbative effect of this electrostatic potential in the system, which causes a triangular-like downward bending of the bulk electronic bands close to the surface by almost $250 \mathrm{meV}$ (Fig. 2b). Combined with the large potential step at the material/vacuum interface, this creates a near-surface quantum well for electrons. The bulk conduction band states are consequently restructured into ladders of multiple subband states ${ }^{7,27,28}$. Our layer-resolved calculations (Fig. 2) show that 
a

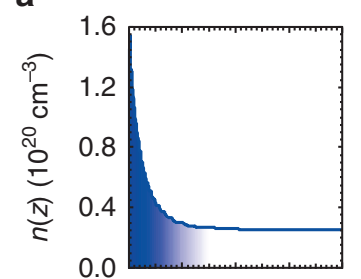

b

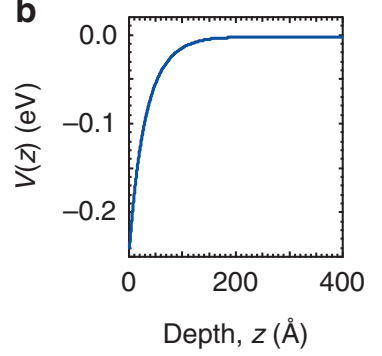

c

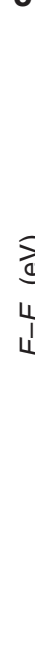

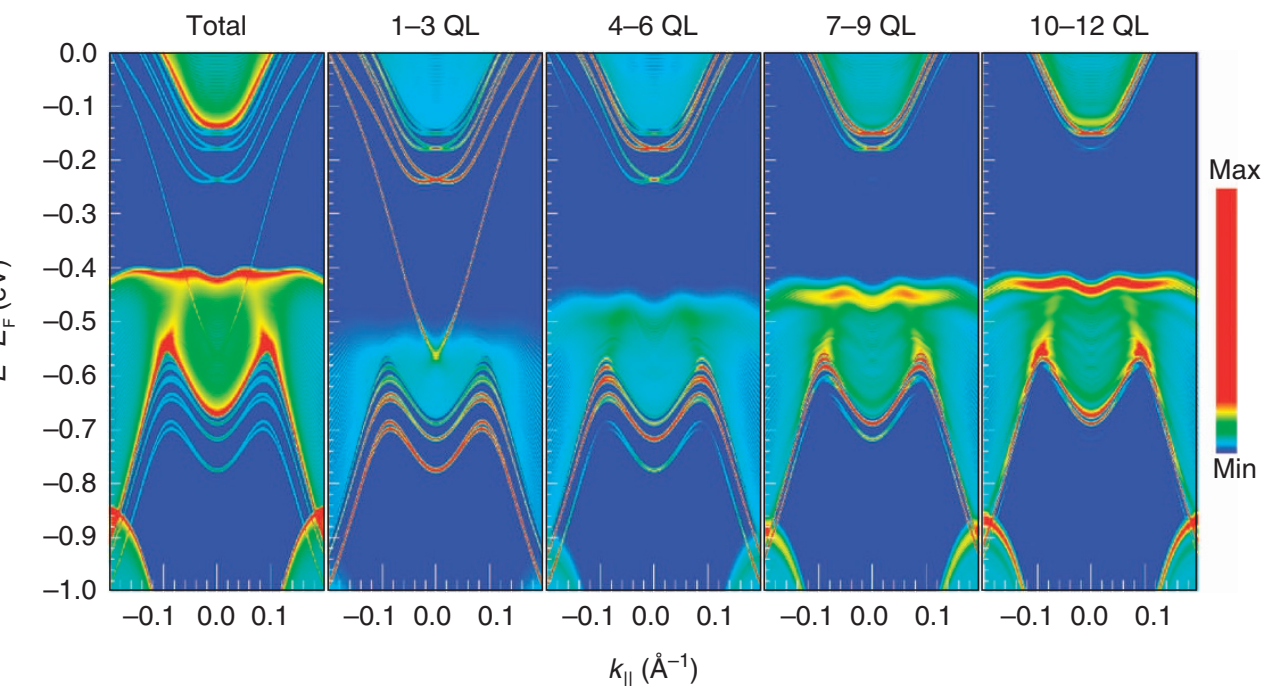

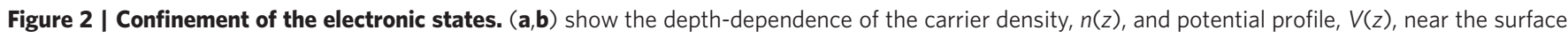
of $\mathrm{Bi}_{2} \mathrm{Se}_{3}$, respectively. The blue shading schematically represents the spatial extent of the surface-localized 2DEG. (c) Quintuple-layer (QL) projected

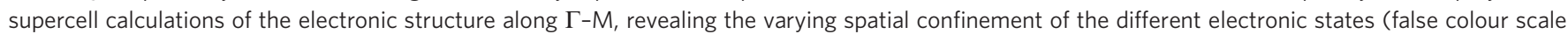
shown to right).

the lowest such subband, lying deep within the potential well, is localized in the topmost few quintuple layers of the structure: The electrons that populate this subband are free to move parallel to the surface, but are strongly confined perpendicular to it, forming a two-dimensional electron gas (2DEG). This state exhibits a strong Rashba spin-orbit splitting, whose calculated magnitude reproduces our experimental observations. Higher-lying states of the subband ladder are less strongly bound within the surface quantum well and contribute significant weight as far as 10-15 quintuple layers below the sample surface. The smaller spin splitting observed experimentally for these higher-lying states is again reproduced by our model calculations, confirming that the Rashba splitting is driven by the potential gradient of the confining electrostatic potential ${ }^{8}$. Similar to the conduction band $2 \mathrm{DEG}$, a ladder of subbands is also observed in the valence band near the zone centre. These hole-like states become quantum confined between the surface and the upper edge of a projected bulk band gap ${ }^{3,11}$, as shown schematically in Fig. 1a. The lowest of these, as for the topological and lower conduction-band quantum well states, is strongly confined within the first 3-4 quintuple layers below the surface. We also find a small spin splitting of these valence band quantum well states, which is not resolved experimentally. Its location, around the top of their M-shaped dispersions, indicates a subtle interplay of spin-orbit interactions between the valence and conduction band subbands ${ }^{29}$. Thus, a hierarchy of electronic states in both energy and spatial extent is created, spanning from the two-dimensional limit of the TSS and lowest subbands of the conduction and valence bands to the three-dimensional continuum (Fig. 1a).

Three-dimensional spin texture. Intriguingly, we find an anticorrelation between this electronic dimensionality, and that of the states' spin texture. This is revealed experimentally via an asymmetry in the matrix element for photoemission when the system is excited by right and left circularly polarized light, respectively (Fig. 3a-c). Such circular dichroism has previously been suggested to probe the spin of the isolated TSS, although the microscopic origin of the dichroism has proved controversial ${ }^{30-34}$. Recently, Park et al. ${ }^{31}$ proposed the existence of an unquenched local orbital angular momentum (OAM) of the TSS. They found that this has the same chiral structure as the spin angular momentum (SAM), permitting the well known spin-momentum locking of the TSS, but opposite helicity due to the strong spinorbit coupling. They proposed that the observed dichroism in ARPES measurements of the TSS results due to this orbital texture. Such circular dichroism, therefore, still reveals the spin texture of the system (up to an arbitrary sign), but indirectly via the orbital sector. We therefore employ this to extract the relative spin texture of the entire low-energy electronic structure of the surfaces of topological insulators. We note that, for the relative changes in dichroism between the bands that we study here we can neglect final-state effects, as transitions from all of these closely spaced initial states will go into the same (broad) final state.

Along the $\Gamma-\mathrm{M}$ mirror direction, symmetry requires that the spin, and therefore also the OAM, must lie entirely within the surface plane $\mathrm{s}^{35}$. Our measurements of circular dichroism extracted along this direction (Fig. 1b) are therefore representative of the in-plane spin-orbital texture. The dichroism changes sign upon moving inwards from the outermost Fermi surface sheet of the topological state to the subsequent Fermi surfaces of the lowest Rashba-split subband $\left(E_{0}\right)$ of the $2 \mathrm{DEG}$. This is entirely consistent with the results of spin-resolved photoemission for these states $^{8}$. More extensive analysis (Supplementary Fig. S3) indicates that this trend continues for the next 2DEG subband. Given the left-handed helicity of the $\mathrm{TSS}^{9}$, these measurements therefore indicate an alternating left-right-left-right-left helicity for the spin of consecutive Fermi surface sheets of the TSS and first $\left(E_{0}\right)$ and second $\left(E_{1}\right)$ quantized conduction subbands.

Away from this direction, dichroism of the inner $E_{0}$ and $E_{1}$ 2DEG Fermi surface sheets vary sinusoidally with angle, $\varphi$, around the Fermi surface (shown in Supplementary Fig. S3). This reveals a model Rashba-like ${ }^{36}$ helical spin texture of these states, with the spin-polarized entirely in the surface plane at all energies. However, for the more spatially localized TSS and outermost 2DEG state, the dichroism additionally develops a strong $\sin (3 \phi)$ component, which is maximal along $\Gamma-\mathrm{K}$ and zero along $\Gamma-\mathrm{M}$ (Fig. 1c and Supplementary Fig. S3). This reflects a strong out-of-plane spin polarization close to the Fermi level, which is correlated with hexagonal warping of the electronic states ${ }^{35-39}$, again switches sign between neighbouring states, and decreases with increasing binding energy (Supplementary Fig. S4). 
a

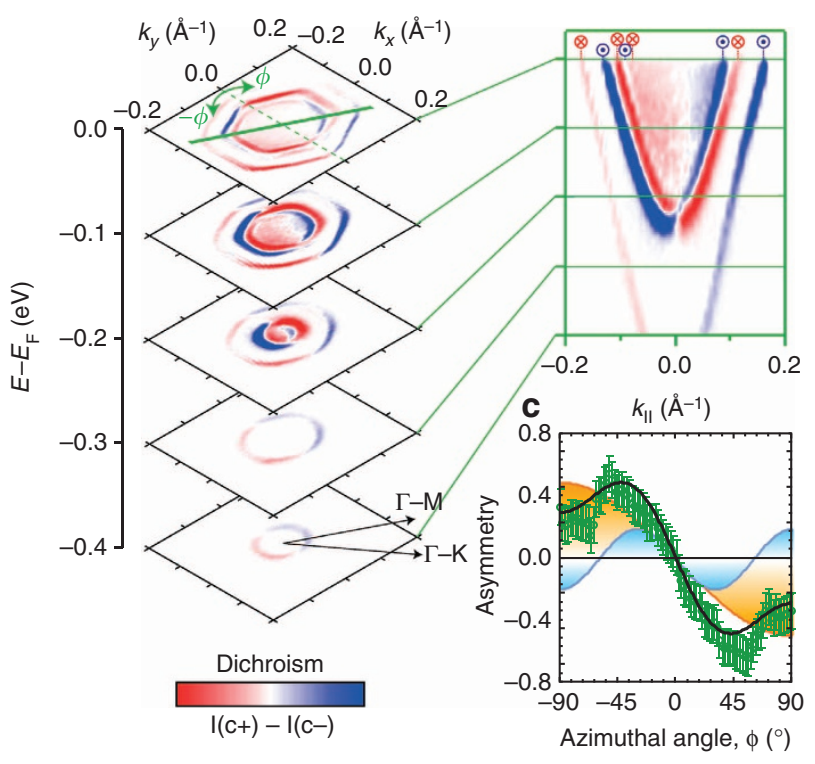

d

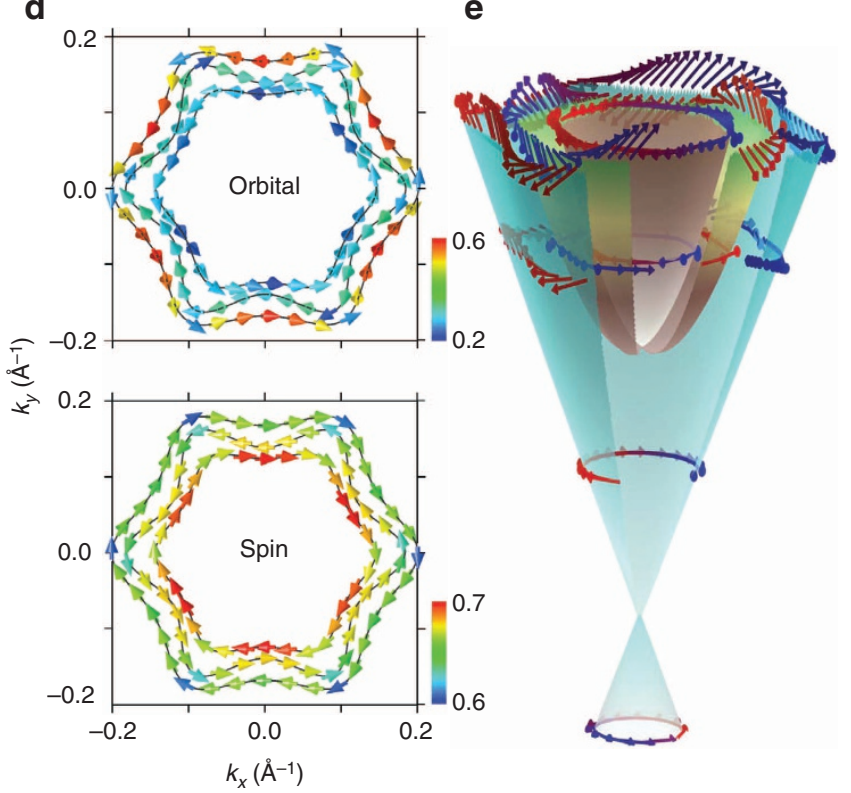

Figure 3 | Three-dimensional orbital and spin texture of the topological and 2DEG states. (a,b) Circular dichroism in constant energy contours and $E$ versus $k$ dispersions measured by ARPES encode the spin-orbital texture of the topological (outer band) and 2DEG (remaining features) states. Circular dichroism along $\Gamma-\mathrm{M}(\mathbf{b})$, extracted along the bold green line shown in $\mathbf{a}$, contains only the in-plane component. This reveals opposite helicity for consecutive Fermi surface sheets, with the spin pointing into $(\otimes)$ or out of $(\odot)$ the plane of the figure. (c) Quantitative analysis of the angular dependence of the dichroism around the Fermi surface, shown for the outer branch of the lowest Rashba-split 2DEG state as a function of aziumthal angle, $\varphi$, from the dashed green line $\left(\Gamma-\mathrm{M}, k_{y}=0\right)$ direction shown in $\mathbf{a}$. Error bars reflect an approximate estimate of the uncertainty in extracting the asymmetry parameter from the experimental measurements, incorporating statistical errors in peak fitting, systematic errors and sample-to-sample variations. The fit to the total dichroism (black line) contains not only a component which varies as $\sin (\phi)$ (yellow), but also a large $\sin (3 \phi)$ contribution (blue), revealing a significant out-of-plane orbital, and therefore also spin, canting. This is consistent with our calculations shown in $\mathbf{d}$ (see also Supplementary Fig. S5), which reveal an alternating helical texture of both the orbital and spin angular momenta (absolute magnitude represented by the false colour scale to the right), but with opposite winding direction. These develop significant out-of-plane components for the larger Fermi surface sheets. In contrast, our calculations and experiment reveal that all other states largely retain the in-plane spin texture characteristic of classic Rashba systems all the way up to the Fermi energy. Together, this leads to a rich three-dimensional spin-texture of the surface electronic structure of topological insulators, as summarized by the arrows on the TSS (blue) and lowest Rashba-split (brown-green) subband of the 2DEG in $\mathbf{e}$.

These findings are fully supported by our tight-binding supercell calculations (Fig. 3d; Supplementary Fig. S5), where we find that the OAM and SAM exhibit an alternating helical structure for consecutive Fermi surface sheets, both also developing increasing out-of-plane components with hexagonal warping. The local OAM, therefore, similar to the spin texture, does lie purely in the surface plane at $k$-points along $\Gamma-\mathrm{M}$, whereas away from this high-symmetry direction it gradually attains a net out-of-plane component, which is maximal along $\Gamma-\mathrm{K}$. However, the OAM helicity of each band is opposite to that of its SAM. This not only confirms that the TSS possesses intricately related OAM and SAM structures, but also reveals that the nontrivial topology drives the TSS and 2DEG states to have a novel alternating helical OAM texture. Thus, not only does the band bending promote the creation of additional Fermi surface sheets, it also leads to complex band- and binding-energy-dependent three-dimensional orbital- and spin textures of the resulting electronic system, as summarized for the spin in Fig. 3e. Together, this will cause significant complications for the interpretation of spin-dependent transport experiments, and inter-band processes will weaken the topological protection against backscattering commonly assumed for the TSS of these compounds. Nonetheless, we note that our resolution limited linewidths of $\lesssim 0.006 \AA^{-1}\left(\lesssim 0.13^{\circ}\right)$ full width at half maximum indicate that the intrinsic scattering remains relatively weak for all Fermi surface sheets.
Surface-bulk connectivity. The complex spin texture of these states also raises questions over their interplay. For any system with timereversal symmetry, the electronic states must be spin-degenerate at time-reversal invariant momenta, even though a breaking of inversion symmetry by the confining potential and surface allows a lifting of the spin degeneracy at arbitrary $k$-points. The hexagonal Brillouin zone of the $\mathrm{Bi}_{2} \mathrm{Se}_{3}$ surface contains time-reversal invariant $k$-points at the zone centre $(\Gamma$ point) and at the sidecentre (M) point. Figure 4a shows the electronic structure calculated along the entire $\Gamma-\mathrm{M}$ direction. At the $\Gamma$ point, each Rashba-split pair of 2DEG states clearly become degenerate. For the TSS, this initially appears to be complicated by the presence of bulk states, with the Dirac point looking to be buried deep in the bulk valence band. However, locally, the TSS is still situated within a projected band gap of the (bent) bulk bands, and so remains a well-defined surface state (unlike the 2DEG states that are located within, and indeed derived from, the projected bulklike bands). Our layer-projected calculations (Fig. 2) show that the Kramer's degeneracy of the TSS is preserved in the presence of the band-bending potential, contrary to previous suggestions of a band gap opening in the surface spectrum at the Dirac point ${ }^{13,17}$.

At the M-point, our calculations reveal that the TSS connects to the outer branch of the lower 2DEG state. Assuming a spindown state for the TSS, the pairing 2DEG state has no other choice but to be spin-up. Its spin-down partner must then 
a

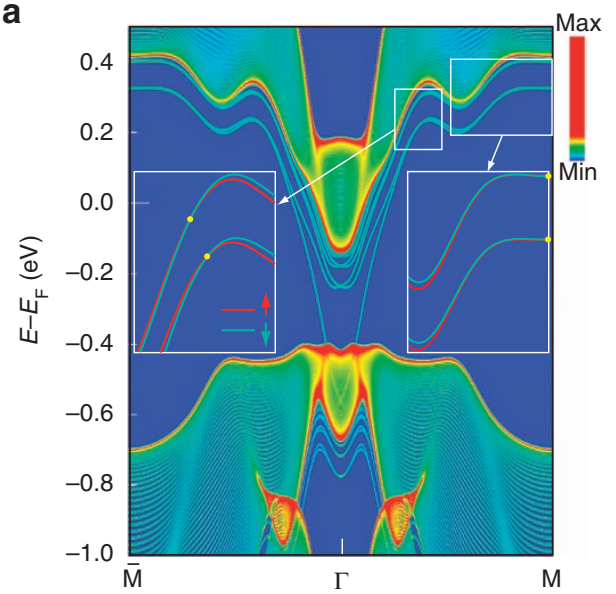

b

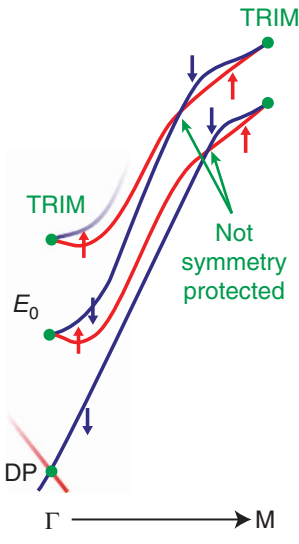

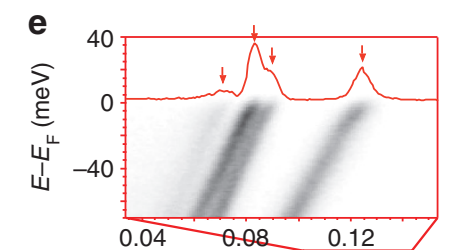

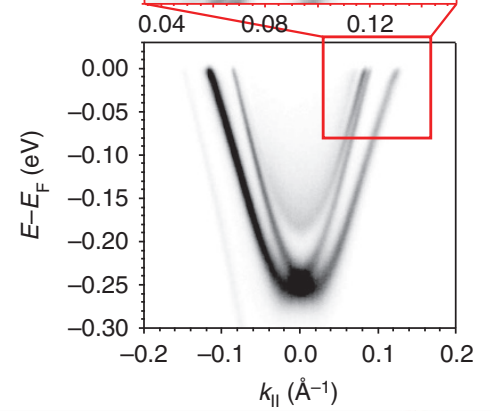

C Topologically

d TI surface quantum well
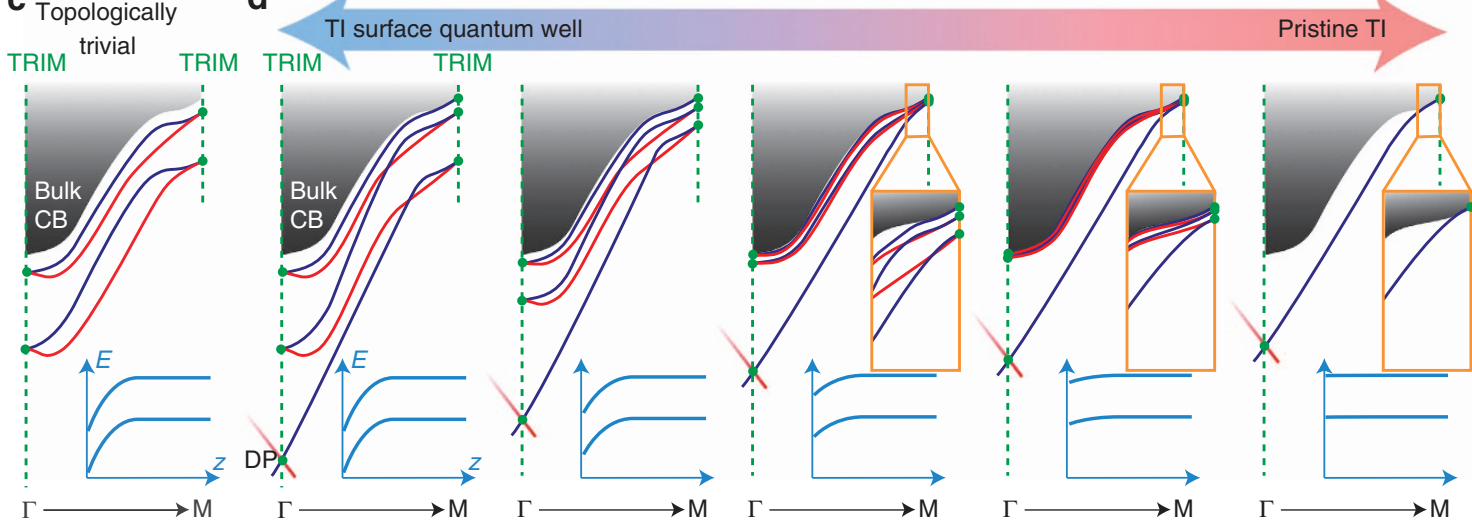

Figure 4 | Connectivity of the surface electronic spectrum. (a) Full electronic structure calculation along the $\Gamma-M$ direction. At the $\Gamma$ point, Kramer's degeneracy requires that the TSS is spin-degenerate (the Dirac point, DP) and each 2DEG state is degenerate with its spin-split pair. At the M-point, this degeneracy occurs between the topological state and outer branch of the lowest 2DEG state, and then between the inner and outer branch of each successive pair of 2DEG states, as shown schematically in $\mathbf{b}$. This forces a divergence of the spin-up (red, up arrows) and spin-down (blue, down arrows) branches of each subband at high wavevectors, in contrast to topologically trivial Rashba systems (c). (d) Schematic evolution of the band connectivity to the bulk of topological insulators as a function of surface band bending (inset), with degeneracy between spin-up (red) and spin-down (blue) bands always maintained at the $\Gamma$ and $M$ points. Between these time-reversal invariant momenta (TRIM), no fundamental symmetry dictates degeneracy of the states. Although additional accidental degeneracies can occur $(\mathbf{a}, \mathbf{b})$, our high-resolution ARPES measurements (e) reveal that, away from the zone centre, all bands remain nondegenerate up until at least the Fermi level. This is clearly observed in a magnified view near the Fermi level (red region), where four distinct peaks arising from spin-split 2DEG states (identified by small arrows) can be resolved in the Fermi level momentum distribution curve.

become connected with the outer branch of the second subband to satisfy TR symmetry for these states, thereby dictating the overall up-down-up-down-up spin configuration discussed above. Fundamentally, this pair-exchange follows from the nontrivial $Z_{2}$ topology of the bulk band structure. This requires that the TSS must connect from the bulk valence to conduction bands. Now, however, the original bulk bands have become quantized into two-dimensional subband states in the vicinity of the surface, and our calculations indicate that it is to these subbands which the topological state connects.

If the band bending is reduced (Fig. 4d), the subband states will become split-off less far below the bulk conduction bands, while their Rashba spin splitting will simultaneously decrease. This will consequently reduce the relative energy separation of the multiple band connectivities at the M-point. Extending this continuously to the limit of no band bending, the subbands evolve into the lower bulk conduction band states, while the zone-edge connectivities merge into a single degeneracy at the M-point between the topological state and the bulk electronic continuum. This clarifies how the surface-bulk connectivity occurs in the idealized case of topological insulators with a pristine surface and no band bending. In the more realistic case where band bending is present, however, the surface-bulk connectivity is mediated via the quantum-well states. This requires the outer and inner branches of the spin-split subbands to diverge from each other at large $k$-vectors, in stark contrast to conventional Rashba systems ${ }^{41}$ in which the two spin channels merge again at zone-edge time-reversal invariant momenta (Fig. 4c). In topologically trivial surface systems where giant Rashba effects have been observed, the spin splitting depends sensitively on details of the near-surface atomic coordinates relative to the surface potential step ${ }^{42}$. In contrast, because of the subband connectivity, the magnitude of the Rashba splitting in a topological insulator 2DEG is directly linked to the subband separation. The possibility to control the 2DEG spin splitting electrostatically via the band bending is therefore intrinsic to topological insulator 2DEGs, rendering these systems ideal candidates for applications in spintronics.

Hexagonal warping of the electronic states can additionally induce accidental degeneracies along high-symmetry lines ${ }^{43,44}$, as seen in Fig. 4. However, as these are not protected by any 
fundamental symmetry, introduction of perturbations in the system can shift, or even completely remove, such accidental degeneracies. Experimentally, the inner branch of the lower 2DEG state and outer branch of the upper 2DEG state can be seen to approach each other at low binding energies (Fig. 4d), consistent with their proximity to the accidental crossing predicted in our calculations. However, contrary to previous reports ${ }^{13,17}$, our high-resolution measurements reveal that the occupied bands remain nondegenerate and exhibit rather parallel dispersion close to the Fermi level, which could be indicative of an avoided crossing.

\section{Discussion}

The electronic structure that we consider here represents the generic surface electronic structure of topological insulators under realistic conditions, such as exposure to atmosphere ${ }^{12,21}$, or in desired devices such as the (spin-)transistor ${ }^{8}$. We have shown that this entire surface electronic system can be accurately described, considering only a single modification of the pristine crystal-a near-surface electrostatic potential variation. The quantitative agreement of our parameter-free tight-binding supercell calculations to our photoemission measurements demonstrates that such a band bending alone is sufficient to induce the entire experimentally measured complex electronic structure of topological insulator surfaces. We stress that our approach does not require any additional modifications beyond an electrostatic potential, such as a huge increased van der Waals gap size $e^{15,16,18,20}$, intercalation ${ }^{45}$, other artificial detachments of a finite number of crystal layers ${ }^{14}$, or the formation of new TSSs ${ }^{13}$.

Despite its conceptual simplicity, the band bending has dramatic consequences for the surface electronic structure. Driven by an emergent role of quantum size effects, it leads to a rich surface electronic system simultaneously comprising both electron- and hole-like subbands of varying spatial confinement and with intertwined three-dimensional spin textures. We have shown how these subbands mediate connectivity of the bulk bands and the TSS, one of the defining properties of topological insulators, through a series of band degeneracies required by time-reversal symmetry, and further identify possible accidental degeneracies, which we do not observe experimentally. Understanding whether interactions not included in our theoretical treatment have a role in avoiding such accidental degeneracies will be crucial to unravel surface-bulk coupling in these materials, and the role of interactions in limiting mobilities of spinpolarized surface transport in topological insulators.

\section{Methods}

Theory. Relativistic electronic structure calculations were carried out within the context of density functional theory using the Perdew-Burke-Ernzerhof exchangecorrelation functional ${ }^{46}$ and the augmented plane wave plus atomic orbitals method as implemented in the WIEN2K program ${ }^{47}$. For all the atoms, the muffintin radius $R_{\mathrm{MT}}$ was set to $2.5 \mathrm{Bohr}$ and the maximum modulus of reciprocal vectors $K_{\max }$ was chosen such that $R_{\mathrm{MT}} K_{\max }=7.0$. The primitive cell was considered to be hexagonal (space group: $\mathrm{R} \overline{3} \mathrm{~m}$ ) with lattice parameters and atomic positions taken from experiment ${ }^{48}$. The corresponding Brillouin zone was sampled using a $10 \times 10 \times 3 \mathrm{k}$-mesh. To simulate the effect of band bending, a 120 qunituple-layer tight-binding supercell Hamiltonian was constructed by downfolding the augmented plane wave plus atomic orbitals Hamiltonian using maximally localized Wannier functions $s^{49-51}$. We chose valence $p$ orbitals of Bi and Se as the projection centres of the Wannier functions. The bending potential was obtained by solving the coupled Poisson-Schrödinger equation ${ }^{26}$, assuming a static dielectric constant of 70 (ref. 8). The resulting potential was then added to the onsite terms of the tight-binding supercell Hamiltonain. The (quintuple-layer-projected) surface band structure was eventually obtained by diagonalizing the supercell Hamiltonian (and projecting the resulting eigenstates onto the maximally localized Wannier functions of respective surface quintuple layers).

Experiment. $\mathrm{Bi}_{2} \mathrm{Se}_{3}$ crystals were prepared by reacting high-purity elements $(5 \mathrm{~N})$ of Bi and Se in sealed, evacuated quartz tubes at $850^{\circ} \mathrm{C}$ for 2 days, followed by cooling at $2-3^{\circ} \mathrm{Ch}^{-1}$ to $650^{\circ} \mathrm{C}$. The crystals were annealed at this temperature for 7 days before quenching to room temperature. ARPES measurements were performed at $10 \mathrm{~K}$ using circularly polarized light with photon energies of $20-30 \mathrm{eV}$ and a Scienta R4000 hemispherical analyser at the SIS beamline of the Swiss Light Source. Surface doping was achieved by depositing potassium at room temperature on freshly cleaved samples from a properly outgassed SAES K getter source. The sample was then immediately transferred to the low-temperature manipulator for ARPES measurements.

\section{References}

1. Hasan, M. Z. \& Kane, C. L. Colloquium: topological insulators. Rev. Mod. Phys. 82, 3045-3067 (2010).

2. Kane, C. L. \& Mele, E. J. $Z_{2}$ topological order and the quantum spin hall effect. Phys. Rev. Lett. 95, 146802 (2005).

3. Zhang, $\mathrm{H}$. et al. Topological insulators in $\mathrm{Bi}_{2} \mathrm{Se}_{3}, \mathrm{Bi}_{2} \mathrm{Te}_{3}$ and $\mathrm{Sb}_{2} \mathrm{Te}_{3}$ with a single Dirac cone on the surface. Nat. Phys 5, 438-442 (2009).

4. Xia, Y. et al. Observation of a large-gap topological-insulator class with a single Dirac cone on the surface. Nat. Phys 5, 398-402 (2009).

5. Chen, Y. L. et al. Experimental realization of a three-dimensional topological insulator, $\mathrm{Bi}_{2} \mathrm{Te}_{3}$. Science 325, 178-181 (2009).

6. Scanlon, D. O. et al. Controlling bulk conductivity in topological insulators: key role of anti-site defects. Adv. Mater 24, 2154-2158 (2012).

7. Bianchi, M. et al. Coexistence of the topological state and a two-dimensional electron gas on the surface of $\mathrm{Bi}_{2} \mathrm{Se}_{3}$. Nat. Commun. 1, 128 (2010).

8. King, P. D. C. et al. Large tunable Rashba spin splitting of a two-dimensional electron gas in $\mathrm{Bi}_{2} \mathrm{Se}_{3}$. Phys. Rev. Lett 107, 096802 (2011).

9. Hsieh, D. et al. A tunable topological insulator in the spin helical Dirac transport regime. Nature 460, 1101-1105 (2009).

10. Noh, H. J. et al. Spin-orbit interaction effect in the electronic structure of $\mathrm{Bi}_{2} \mathrm{Te}_{3}$ observed by angle-resolved photoemission spectroscopy. Europhys. Lett 81, 57006 (2008).

11. Bianchi, M., Hatch, R. C., Mi, J., Iversen, B. B. \& Hofmann, P. Simultaneous quantization of bulk conduction and valence states through adsorption of nonmagnetic impurities on $\mathrm{Bi}_{2} \mathrm{Se}_{3}$. Phys. Rev. Lett. 107, 086802 (2011).

12. Benia, H. M., Lin, C., Kern, K. \& Ast, C. R. Reactive chemical doping of the $\mathrm{Bi}_{2} \mathrm{Se}_{3}$ topological insulator. Phys. Rev. Lett. 107, 177602 (2011).

13. Wray, L. A. et al. A topological insulator surface under strong Coulomb, magnetic and disorder perturbations. Nat. Phys. 7, 32-37 (2011).

14. Zhu, Z.-H. et al. Rashba spin-splitting control at the surface of the topological insulator $\mathrm{Bi}_{2} \mathrm{Se}_{3}$. Phys. Rev. Lett. 107, 186405 (2011).

15. Eremeev, S. V., Menshchikova, T. V., Vergniory, M. G. \& Chulkov, E. V. New interpretation of the origin of 2DEG states at the surface of layered topological insulators. Preprint at http://arXiv.org/abs/1107.3208 (2011).

16. Menshchikova, T., Eremeev, S. \& Chulkov, E. On the origin of two-dimensional electron gas states at the surface of topological insulators. JETP Lett. 94, 106-111 (2011).

17. Wray, L. A. et al. Electron dynamics in topological insulator based semiconductor-metal interfaces (topological $p-n$ interface based on $\mathrm{Bi}_{2} \mathrm{Se}_{3}$ class). Preprint at http://arXiv.org/abs/1105.4794 (2011).

18. Ye, M. et al. Relocation of the topological surface state of $\mathrm{Bi}_{2} \mathrm{Se}_{3}$ beneath the surface by Ag intercalation. Preprint at http://arXiv.org/abs/1112.5869 (2011).

19. Valla, T., Pan, Z.-H., Gardner, D., Lee, Y. S. \& Chu, S. Photoemission spectroscopy of magnetic and nonmagnetic impurities on the surface of the $\mathrm{Bi}_{2} \mathrm{Se}_{3}$ topological insulator. Phys. Rev. Lett. 108, 117601 (2012).

20. Vergniory, M. G., Menshchikova, T. V., Eremeev, S. V. \& Chulkov, E. V. Ab initio study of $2 \mathrm{DEG}$ at the surface of topological insulator $\mathrm{Bi}_{2} \mathrm{Te}_{3}$. JETP Lett. 95, 230-235 (2012).

21. Chen, C. et al. Robustness of topological order and formation of quantum well states in topological insulators exposed to ambient environment. Proc. Nat. Acad. Sci. 109, 3694 (2012).

22. Zhang, F., Kane, C. L. \& Mele, E. J. Surface states of topological insulators. Phys. Rev. B 86, 081303(R) (2012).

23. King, P. D. C. et al. Surface electron accumulation and the charge neutrality level in $\mathrm{In}_{2} \mathrm{O}_{3}$. Phys. Rev. Lett. 101, 116808 (2008).

24. Mönch, W. Semiconductor Surfaces and Interfaces (Springer, Berlin, 2001).

25. Lin, H. et al. Half-Heusler ternary compounds as new multifunctional experimental platforms for topological quantum phenomen. Nat. Mater. 9, 546-549 (2010)

26. King, P. D. C., Veal, T. D. \& McConville, C. F. Nonparabolic coupled PoissonSchrödinger solutions for quantized electron accumulation layers: band bending,charge profile,and subbands at InN surfaces. Phys. Rev. B 77, 125305 (2008).

27. King, P. D. C. et al. Surface band-gap narrowing in quantized electron accumulation layers. Phys. Rev. Lett. 104, 256803 (2010).

28. Meevasana, W. et al. Creation and control of a two-dimensional electron liquid at the bare $\mathrm{SrTiO}_{3}$ surface. Nat. Mater 10, 114-118 (2011).

29. Bahramy, M. S., Arita, R. \& Nagaosa, N. Origin of giant bulk Rashba splitting: application to BiTeI. Phys. Rev. B 84, 041202(R) (2011). 
30. Wang, Y. H. et al. Observation of a warped helical spin texture in $\mathrm{Bi}_{2} \mathrm{Se}_{3}$ from circular dichroism angle-resolved photoemission spectroscopy. Phys. Rev. Lett. 107, 207602 (2011).

31. Park, S. R. et al. Chiral orbital-angular momentum in the surface states of $\mathrm{Bi}_{2} \mathrm{Se}_{3}$. Phys. Rev. Lett. 108, 046805 (2012).

32. Ishida, Y. et al. Common origin of the circular-dichroism pattern in angleresolved photoemission spectroscopy of $\mathrm{SrTiO}_{3}$ and $\mathrm{Cu}_{x} \mathrm{Bi}_{2} \mathrm{Se}_{3}$. Phys. Rev. Lett 107, 077601 (2011).

33. Mirhosseini, H. \& Henk, J. Spin texture and circular dichroism in photoelectron spectroscopy from the topological insulator $\mathrm{Bi}_{2} \mathrm{Te}_{3}$ : first-principles photoemission calculations. Phys. Rev. Lett. 109, 036803 (2012).

34. Jung, W. et al. Warping effects in the band and angular-momentum structures of the topological insulator $\mathrm{Bi}_{2} \mathrm{Te}_{3}$. Phys. Rev. B 84, 245435 (2011).

35. Fu, L. Hexagonal warping effects in the surface states of the topological insulator $\mathrm{Bi}_{2} \mathrm{Te}_{3}$. Phys. Rev. Lett 103, 266801 (2009).

36. Bychkov, Y. A. \& Rashba, E. I. Properties of a $2 \mathrm{D}$ electron gas with lifted spectral degeneracy. JETP Lett 39, 78 (1984).

37. Yazyev, O. V., Moore, J. E. \& Louie, S. G. Spin polarization and transport of surface states in the topological insulators $\mathrm{Bi}_{2} \mathrm{Se}_{3}$ and $B i_{2} \mathrm{Te}_{3}$ from first principles. Phys. Rev. Lett. 105, 266806 (2010).

38. Souma, S. et al. Direct measurement of the out-of-plane spin texture in the Dirac-cone surface state of a topological insulator. Phys. Rev. Lett. 106, 216803 (2011).

39. $\mathrm{Xu}, \mathrm{S} .-\mathrm{Y}$. et al. Topological phase transition and texture inversion in a tunable topological insulator. Science 332, 560-564 (2011).

40. Frantzeskakis, E. \& Grioni, M. Anisotropy effects on rashba and topological insulator spin-polarized surface states: a unified phenomenological description. Phys. Rev. B 84, 155453 (2011).

41. Ishizaka, K. et al. Giant Rashba-type spin splitting in bulk BiTeI. Nat. Mater 10, 521-526 (2011).

42. Ast, C. R. et al. Giant spin splitting through surface alloying. Phys. Rev. Lett. 98, 186807 (2007)

43. Bahramy, M. S. et al. Emergence of non-centrosymmetric topological insulating phase in BiTeI under pressure. Nat. Commun 3, 679 (2012).

44. Basak, S. et al. Spin texture on the warped Dirac-cone surface states in topological insulators. Phys. Rev. B 84, 121401(R) (2011).

45. Bianchi, M. et al. Robust surface doping of $\mathrm{Bi}_{2} \mathrm{Se}_{3}$ by rubidium intercalation. ACS Nano. 6, 7009-7015 (2012).

46. Perdew, J. P., Burke, K. \& Ernzerhof, M. Generalized gradient approximation made simple. Phys. Rev. Lett. 77, 3865-3868 (1996).

47. Blaha, P. et al. WIEN2K package, Version 10.1 (2010); available at http:// www.wien $2 \mathrm{k}$.at.
48. Vicente, C. P. et al. X-ray diffraction and ${ }^{119} \mathrm{Sn}$ Mössbauer spectroscopy study of new phase in the $\mathrm{Bi}_{2} \mathrm{Se}_{3}-\mathrm{SnSe}$ system: $\mathrm{SnBi}_{4} \mathrm{Se}_{7}$. Inorg. Chem. 38, 2131-2135 (1999).

49. Souza, I. et al. Maximally localized Wannier functions for entangled energy bands. Phys. Rev. B 65, 035109 (2001).

50. Mostofi, A. A. et al. Wannier90: a tool for obtaining maximally localized Wannier functions. Comp. Phys. Commun. 178, 685-699 (2008).

51. Kuneš, J. et al. WIEN2WANNIER: from linearized augmented plane waves to maximally localized Wannier functions. Comp. Phys. Commun. 181, 1888-1895 (2010).

\section{Acknowledgements}

We are grateful for support from the UK EPSRC, the ERC and the Japan Society for the Promotion of Science (JSPS) through the 'Funding Program for World-Leading Innovative R\&D on Science and Technology (FIRST Program)', initiated by the council for Science and Technology Policy (CSTP). This work was also supported by Grant-in-Aids for Scientific Research (No. 24224009) from the Ministry of Education, Culture, Sports, Science and Technology (MEXT) of Japan, and Strategic International Cooperative Program (Joint Research Type) from Japan Science and Technology Agency.

\section{Author contributions}

MSB developed the tight-binding model with assistance from RA, and performed the tight-binding model calculations. PDCK, ADLT and FB performed the ARPES measurements. PDCK analysed the ARPES data and performed the Poisson-Schrödinger calculations. JC, MS and LP maintained the ARPES endstation at the Swiss Light Source and assisted with the ARPES measurements. PH contributed preliminary measurements and discussions. GB grew the samples. PDCK, MSB and FB wrote the paper with input from all co-authors. All authors discussed the interpretation of the results and commented on the final manuscript. PDCK, MSB, NN and FB were responsible for overall project planning, infrastructure and direction.

\section{Additional information}

Supplementary Information accompanies this paper on http://www.nature.com/ naturecommunications

Competing financial interests: The authors declare no competing financial interests.

Reprints and permission information is available online at http://npg.nature.com/ reprintsandpermissions/

How to cite this article: Bahramy, M.S. et al. Emergent quantum confinement at topological insulator surfaces. Nat. Commun. 3:1159 doi: 10.1038/ncomms2162 (2012). 